\title{
Rediseño curricular por competencias: experiencia en la formación inicial universitaria en Chile
}

\author{
Verónica Díaz-Quezada, Álvaro Poblete-Letelier y Mónica Gallardo-González
}

\section{RESUMEN}

Este trabajo describe la experiencia del rediseño curricular por competencias de la carrera de Pedagogía en Matemática y Computación en la Universidad de Los Lagos, Chile. Se detalla el contexto y la totalidad de las fases del rediseño. Los resultados concretos surgieron del proceso de autoevaluación de la carrera, que en la actualidad cuenta con un nuevo plan de estudios, un perfil de egreso adecuado al currículo de formación basado en competencias y un importante mejoramiento en las tasas de titulación, además de fortalecimiento de habilidades fundamentales de los estudiantes, certificadas por la Comisión Nacional de Acreditación.

Palabras clave: evaluación de la calidad, educación superior, rediseño curricular, enfoque por competencias, formación docente matemáticas, Chile.

Verónica Díaz-Quezada

mvdiaz@ulagos.cl

Chilena. Doctora en Educación con Especialización en Matemática, Universidad Academia Humanismo Cristiano, Chile; Magister en Evaluación Educacional, Universidad de Playa Ancha de Ciencias de la Educación, Chile. Profesora Titular, Departamento Ciencias Exactas, Universidad de Los Lagos, Chile. Temas de investigación: educación matemática/didáctica de la matemática, resolución de problemas en matemáticas, competencias en matemáticas, evaluación educativa.

\section{Álvaro Poblete-Letelier}

apoblete@ulagos.cl Chileno. Doctor en Didáctica de la Matemática, Universitè Laval, Canadá; Magister en Enseñanza de la Matemática, Universidad de Campinas, Brasil. Profesor Titular, Departamento Ciencias Exactas, Universidad de Los Lagos, Chile. Temas de investigación: educación matemática/didáctica de la matemática, resolución de problemas en matemáticas, competencias en matemáticas.

Mónica Gallardo-González

mgallardo@ulagos.cl

Chilena. Master en Ingeniería de Medios para la Educación, Universidad de Poitiers, Francia; Universidad Técnica de Lisboa, Portugal; Universidad Nacional de Educación a Distancia, España. Profesora Asistente, Departamento Ciencias Exactas, Universidad de Los Lagos, Chile. Temas de investigación: informática educativa, tecnología educativa. 


\section{Redefinição curricular por competências: experiência na formação inicial universitária no Chile}

\section{RESUMO}

Este trabalho descreve a experiência e seus resultados da redefinição curricular por competências do curso de Pedagogia em Matemática e Computação na Universidade de Los Lagos, Chile. Se detalha o contexto e a totalidade das fases da redefinição. Os resultados concretos surgiram do processo de autoavaliação do curso, que na atualidade conta com um novo plano de estudo, um perfil de formandos adequado ao currículo de formação baseado em competências e um importante melhoramento nas taxas de conclusão do curso, além do fortalecimento de habilidades fundamentais dos estudantes, certificadas pela Comisión Nacional de Acreditación.

Palavras chave: avaliação da qualidade, educação superior, redefinição curricular, enfoque por competências, formação docente matemáticas, Chile.

\section{Redesigning curriculum by competencies: experience in initial university training in Chile}

\section{ABSTRACT}

This work describes the experience and results of redesigning curriculum by competences for the degree in Pedagogy of Mathematics and Computing at the University of Los Lagos, Chile. The context and the totality of the phases of the redesign are detailed. The concrete results emerged from the degree's self-assessment process, which now has a new study plan, a graduation profile appropriate to the competencybased curriculum, and a significant improvement in degree rates, in addition to strengthening students' fundamental skills, certified by the National Accreditation Commission.

Key words: quality assessment, higher education, curriculum redesign, competency-based approach, mathematics teacher training, Chile. 


\section{Introducción}

El sistema de educación superior se encuentra en un periodo de redefinir el proceso de enseñanza, con el propósito de promover experiencias significativas de aprendizaje para los estudiantes. En este contexto se firmó en 2000 la Declaración de Bolonia, que propuso un marco común de enseñanza y aprendizaje en el sistema de educación superior europeo. Producto de esta declaración, se instituyó el Tuning Educational Structures in Europe (2006), conocido como Proyecto Tuning (Cisterna, Soto y Rojas, 2016). Como consecuencia, surge el Proyecto Tuning América Latina (2007) con un enfoque de educación basada en competencias, en un contexto globalizado que responde a nuevas necesidades de la sociedad actual, con una educación más flexible, abierta y estrechamente relacionada con los sectores productivos y que ha originado grandes transformaciones en la educación a nivel mundial (Álvarez, 2011; Muñoz-Osuna, Medina-Rivilla y Guillén-Lúgigo, 2016).

En la actualidad, la Organización para la Cooperación y el Desarrollo Económicos (OCDE), con el objetivo de ampliar el conocimiento comparable sobre las habilidades y competencias, trabaja en la Evaluación de los Resultados de Aprendizaje en la Educación Superior (AHELO). A diferencia de otras evaluaciones de la OCDE, el proyecto AHELO trabaja para evaluar lo que los estudiantes de la educación superior saben y pueden hacer tras titularse, centrándose en las habilidades genéricas comunes a todos los estudiantes, como el pensamiento crítico, el razonamiento analítico, la resolución de problemas y la comunicación escrita; y en habilidades específicas para las áreas de economía e ingeniería (OCDE, 2012).

Con preocupación similar, en Chile se implementaron evaluaciones en las carreras de pedagogía de la educación superior, como una forma de garantizar capacidades instaladas en calidad en la formación terciaria, lo que ha dado lugar a que existan diversas entidades interesadas en verificarlas (Icarte y Labate, 2016).
De acuerdo con el informe de OCDE (2013), a lo largo de los últimos 25 a 30 años, Chile ha estado a la vanguardia de Latinoamérica en diversas iniciativas de la educación superior, como los procesos de acreditación y aseguramiento de la calidad, y es el Estado el responsable de la acreditación de las propias instituciones, como también de las carreras de pregrado y posgrado, a través de la Comisión Nacional de Acreditación (GNA).

En este contexto, abordamos un rediseño curricular de la carrera de Pegagogía en Matemática y Computación, logrado con base en un Modelo de Competencias Profesionales de Matemática (MCPM), y al desarrollo de etapas, que incluyeron perfeccionamiento docente, un enfoque didáctico curricular, asesorías técnicas, definición del perfil de egreso y definición del plan de estudios, las cuales se detallan in extenso en este artículo.

\section{Aseguramiento de la calidad}

El clima de preocupación por la calidad de la formación en pedagogía, explica el desarrollo de una serie de políticas y acciones destinadas a corregir problemas observados, lo que llevó en 2004 al Ministerio de Educación en Chile (MINEDUC) a financiar proyectos concursables destinados a mejorar los procesos de formación docente, y en 2006, con la Ley de Aseguramiento de la Calidad de la Educación Superior, a decretar con carácter obligatorio la acreditación para todas las carreras de pedagogía, lo que implica el cumplimiento de ciertos estándares de calidad y la realización de procesos de evaluación y autoevaluación permanentes, que son verificados por la CNA o por agencias acreditadoras. Desde 2016, esta verificación es realizada sólo por la CNA, organismo que utiliza criterios de calidad, los cuales deben complementarse con el perfil profesional que la propia carrera haya tomado.

Las carreras pueden acreditarse hasta por un máximo de siete años o no acreditarse. Para lograr una certificación de calidad por parte de la GNA, 
una carrera debe evaluar el estado de estos criterios en su propia institución, los que son analizados en dos momentos: la autoevaluación, en donde se evalúa el estado de los criterios de calidad con estudiantes, docentes, egresados y empleadores; en un segundo momento, con una evaluación externa por pares académicos designados por el organismo acreditador. En esta secuencia de acciones, la autoevaluación constituye uno de los pilares del proceso de aseguramiento de la calidad, ya que es fuente de información fundamental para las carreras que desean detectar debilidades y fortalezas, así como también monitorear sus propios avances y generar estrategias de mejora (Rodríguez, Fleet y Delgado, 2009). En este contexto, una manifestación del aseguramiento de la calidad universitaria está dada por los procesos de revisión del currículo de las carreras.

$\mathrm{Al}$ respecto, en la literatura educativa se proponen diversos modelos para el diseño curricular. Autores como Vélez y Terán (2009) hacen referencia al modelo tradicional de Tyler (1960), modelo vínculo sociedad-individuo de Taba (1974), modelo de enfoque práctico de Schwab (1983), modelo prácticoprofesional de Walker (1990) y el diseño curricular de Arnaz (1993). Por otro lado, autores como Riesco (2008), Palominos, Méndez y Barrera (2014), Villa y Poblete (2007) se refieren al modelo de formación por competencias del Espacio Europeo de la Educación Superior (EEES), formulado en 1999, enfocado en desarrollar competencias genéricas y específicas que nacen de requerimientos esenciales de cada profesión (Avendaño et al., 2016).

\section{Modelo curricular por competencias}

Las universidades chilenas aplicaron por varios años el modelo tradicional basado en objetivos (CRUCH, 2012), con deficiencias ante las nuevas exigencias del entorno y sus participantes, generando dificultades como la transmisión de los conocimientos teóricos y el desarrollo de nuevas capacidades del profesional. Estos modelos, según el informe de la OCDE (2009), se han caracterizado por currículos rígidos, programas de formación muy extensos, y métodos de enseñanza y aprendizaje evaluados como tradicionales y centrados en la trasmisión mecánica de contenidos (Guzmán et al., 2015). Como una solución, se incorporó el modelo basado en competencias, que entregaría mayor eficiencia y calidad en el proceso de aprendizaje y daría respuesta a las demandas de la sociedad (Avendaño et al., 2016). Tras más de una década de armonización y homologación de competencias transversales y profesionales en Chile y el resto del mundo (Bustamante et al., 2015), el nuevo modelo de formación basado en competencias modifica la estructura curricular pasando de un enfoque basado en el logro de objetivos a uno basado en la formación de competencias (Tardif, 2003), lo que genera un enfoque pedagógico centrado en el estudiante (Tejada, 2002).

En la definición de competencia, aunque sigue siendo muy discutida, aún no se logra consenso (Fletcher, 1997; Mansfield, 1996; Roe, 2002; Spencer y Spencer, 1993). Para Velasco et al. (2014) la palabra competencia es parte de nuestro lenguaje cotidiano, pero tiene diferentes significados en diferentes campos, tales como académico-psicológico, económico, educativo, etcétera. También hay un análisis crítico del concepto de competencia, que se está utilizando en la educación superior (Lozano et al., 2012).

Le Boterf (2001) señala que las competencias deben definirse más en términos de saber actuar que de saber hacer. Para los autores Daugherty, Davis y Miller (2015) la mayoría coincide en al menos una característica central: la educación basada en competencias se centra en lo que se espera que un estudiante sabe y es capaz de hacer al final del curso o programa, es decir, competencias y lograr el crédito del curso o un título/certificado cuando el estudiante ha demostrado el dominio de estas competencias (Bell y Conklin, 2013; Person, Goble y Bruch, 2014; Porter y Reilly, 2014).

En Estados Unidos no existe un acuerdo generalizado sobre cómo definir la "competencia" en 
comparación con el "dominio" o "resultado de aprendizaje" (Nodine, 2016). La llamada Educación Basada en Competencia (o CBE por sus siglas en inglés) hace hincapié en el dominio de los conceptos discretos, conocimientos y habilidades, independientemente de cuánto tiempo se tarda en lograr tal dominio o cuándo se alcanza el dominio (Kelchen, 2015). Por su parte, Boyatzis (2007) define la competencia como una capacidad o habilidad, referida a un conjunto de conjuntos, relacionados pero diferentes, de comportamiento organizado en torno a un constructo subyacente. Markus, Cooper-Thomas y Allpress (1997) señalaron que la literatura se ha acercado a la competencia de tres maneras diferentes: educación, comportamiento y negocio. Perrenoud (2013) ha señalado que la competencia es el poder de actuar con eficacia en una situación, movilizando y combinando, en tiempo real y de manera pertinente, recursos intelectuales y emocionales.

En América Latina, en la actualidad, el enfoque educativo por competencias ha tomado posesión del campo educativo en todos los niveles: básico, técnico-profesional y universitario (Cuba, 2016). A juicio de Verano-Tacoronte et al. (2016) la inclusión del enfoque por competencias en la enseñanza universitaria está motivada no sólo por lograr la coherencia de todo el sistema educativo, sino también por reorientar el papel actual de la educación superior y fortalecer su vínculo con el mundo laboral, potenciando el desarrollo interdisciplinario del estudiante (Bolívar-Cruz et al., 2011; López-Ruiz, 2011; Nassif, Hanashiro y Torres, 2010).

Tobón (2004), Zabala y Arnau (2007) han definido el concepto de competencia y coinciden en que es un término holístico que incluye habilidades, destrezas y actitudes en un contexto especifico en donde son utilizadas no sólo en forma conceptual y procedimental sino también en forma actitudinal.

Para Tobón (2013) las competencias corresponden a un conjunto de desempeños que evidencian el dominio del saber, saber hacer y saber ser; en diferentes contextos y fomentando su capacidad de resolver problemas complejos en diferentes situaciones. Por su parte Zabala y Arnau (2007) específicamente definen la competencia como la actuación eficiente ante una situación concreta y real, que se da en contexto determinado. Colocan la competencia en relación no sólo con los saberes, sino con el sistema educativo y escolar y con la articulación de propuestas para la formación según las necesidades de los individuos y de la sociedad.

Zabalza (2012) señala que lograr el dominio de una competencia no es sólo saber hacer cosas, sino tener suficientes conocimientos para poder responder de manera fundamentada a cuestiones que son relevantes en la actividad profesional.

\section{Modelo curricular por competencias en matemáticas}

En el ámbito de las matemáticas, en la comunidad internacional el enfoque por competencia, es considerado por la OCDE (2003) como una propuesta educativa que no sólo abarca aprendizaje de contenidos, sino que apunta a la formación de ciudadanos constructivos y con capacidad de reflexión, permitiéndoles identificar y entender el rol que juegan las matemáticas en el mundo (Díaz y Poblete, 2016a). A partir del Proyecto DeSeCo de la OCDE, que se concentró en la elaboración de un listado de competencias-clave, diversos países de la Unión Europea han utilizado el enfoque por competencias, como sustento para sus reformas curriculares de la educación obligatoria (Rychen y Salganik, 2006; OCDE, 2005).

Para autores como Pochulu, Font y Rodríguez (2016), en la última década aumentó el interés por investigar el conocimiento y las competencias que necesitan los profesores de matemáticas para conseguir una enseñanza eficaz (Burger y Starbird, 2005; Wilson, Cooney y Stinson, 2005; Hill et al., 2008). Respecto a las competencias de los profesores de secundaria de matemáticas, Díaz y Poblete (2009a, 2009b, 2015, 2016b) proponen un modelo 
de competencia profesional que incluye competencias generales y especializadas, marcos de contextos de competencias, y dimensiones cualitativas en relación con la concepción de calidad.

\section{Formación inicial docente en matemáticas}

La forma en cómo ha evolucionado la formación de profesores de matemáticas durante los últimos 15 años ha sido objeto de diversos estudios centrados en el análisis de las políticas de formación docente (Cox, Meckes y Bascopé, 2010), en su efectividad para producir conocimiento pedagógico general y de contenido matemático (Ávalos y Matus, 2010) y en la relación entre calidad de las instituciones en que se albergan los programas de formación docente y el nivel de conocimiento de los futuros profesores (Pedraja et al., 2012). También se ha examinado la relación entre formación docente y elección del primer empleo y la distribución de nuevos profesores en los establecimientos educativos según tipo de institución formadora de la que egresó (Paredes et al., 2013; Meckes y Bascopé, 2012). Estos estudios y la percepción de quienes siguen de cerca los procesos de formación docente han alertado respecto a una serie de condiciones problemáticas de la formación inicial docente en Chile (Ávalos, 2014).

\section{Formación docente en matemática en la Universidad de Los Lagos}

Desde sus inicios en 1964 como sede de la Universidad de Chile en Osorno, la Universidad de Los Lagos ha mantenido una tradición en la formación de profesores de educación media, y en particular en Pedagogía en Matemática desde 1967. En 1998 se crea la carrera de Pedagogía en Matemática y Computación con un proyecto curricular por objetivos y centrado en la organización de núcleos temáticos de contenido y sin lograr una adecuada integración entre situaciones de contenido matemático y de didáctica, lo que generó la necesidad de un cambio curricular. Este cambio se hizo efectivo a partir del dictamen del proceso de acreditación anterior de la carrera, en el cual la CNA hizo notar que el perfil de egreso era explícito, sin embargo, su formulación no era suficientemente clara y completa como para orientar la definición del plan de estudios. Además, no se hacía cargo de manera explícita, de las competencias asociadas al otorgamiento del grado de licenciado en Educación. También indicó que si bien la línea de prácticas progresivas estaba bien diseñada y constituía una fortaleza de la carrera, la falta de participación de académicos de jornada completa de la especialidad, reducía su capacidad para retroalimentar el proceso formativo. La formación pedagógica y la formación de especialidad no se encontraban debidamente articuladas, lo que limitaba las posibilidades de integración armónica de los distintos componentes de la formación profesional. Finalmente, indicaron que la progresión de los estudiantes era lenta, debido a la rigidez del currículo y al hecho de que los cursos no se dictaban todos los semestres. El tiempo de egreso era excesivo y las tasas de titulación muy bajas, sin que se observaran acciones concretas para enfrentar estas situaciones. Con todo lo anterior, unido a los deficientes resultados de aprendizaje de los estudiantes, se decidió por un rediseño curricular de la carrera.

\section{Proceso de rediseño curricular}

A través de un proyecto de investigación y desarrollo MECESUP ULA0608 (Poblete y Díaz, 2008), en la carrera de Pedagogía en Matemática y Computación se realizó un rediseño curricular orientado al desarrollo de competencias (Proyecto Tuning América Latina, 2007), tendiente a lograr la calidad de los aprendizajes y con la enseñanza focalizada en la obtención de logros de aprendizaje significativos, en cuanto las ideas se relacionen de un modo sensible (con sentido) con las ideas que ya se tienen.

Desde la perspectiva del rediseño curricular de la Pedagogía en Matemática y Computación, el interés primordial no consiste solamente en que la población 
estudiantil conozca en profundidad su saber disciplinario, su arte y técnica (el lenguaje de la modernidad), sino que además se forme en habilidades y destrezas con perspectiva interdisciplinaria, en valores, en creatividad, en resolución de problemas y en pensamiento crítico. Se pensó en un profesional formado para enfrentar los problemas de su profesión y, además, los problemas de su vida en la sociedad del siglo XXI.

\section{Objetivo}

Este rediseño curricular desde la investigación contempla como objetivo diseñar, elaborar y aplicar un programa curricular de formación basado en competencias, integral y contextualizado, cuya orientación pedagógica promueva aprendizajes significativos que se relacionen con el desarrollo de una base formativa común de desempeño profesional especializado en matemática, en computación e informática y en lo didáctico-pedagógico.

\section{Marco teórico}

El referente teórico general fue el Proyecto Tuning
América Latina (2007), por cuanto propone el concepto de competencias y resultados de aprendizaje como elementos clave para el diseño de los planes de estudio, las metodologías de enseñanza y los procesos evaluativos. Este enfoque responde a una concepción del perfil profesional que favorece el desempeño autónomo, el pensamiento crítico, las habilidades de comunicación, el dominio de otros idiomas, la ética y el autoaprendizaje (Hambur, Rowe y Lucgrew, 2002). En concordancia con el referente teórico, se adoptó el Sistema de Créditos Académicos Transferibles (SCT) que considera la carga real de trabajo del estudiante requerida para completar cada una de las actividades planificadas (Dochy y Nickmans, 2005).

El marco teórico específico se basa en las competencias profesionales que forman parte de un Modelo de Competencias Profesionales de Matemática (MCPM) (Poblete y Díaz, 2004) que fue previamente validado y evaluado a través de la ejecución de un proyecto Fondecyt 1010980, financiado por la Comisión Nacional de Investigación Científica de Chile, que se muestra en la figura 1.

\section{Figura 1. Modelo de Competencias Profesionales de Matemática (MCPM)}

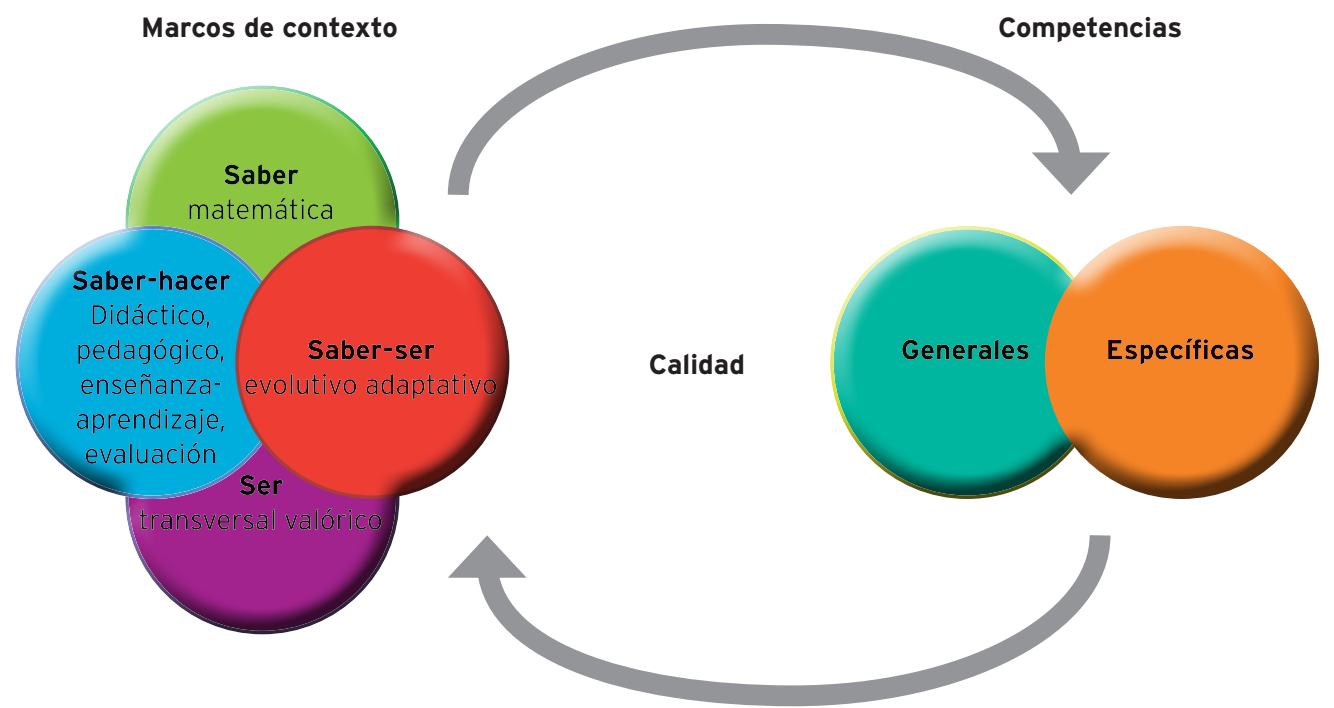

Fuente: Poblete y Díaz (2004). 
En este modelo se definen tanto la competencia del profesor de matemáticas, como la habilidad adquirida efectiva y eficientemente al ejecutar el acto de enseñar matemáticas, relacionada con la calidad, en el sentido de hacer la tarea educativa de formación y hacerla bien. Esto indica que tal habilidad necesariamente integra saberes y conocimientos para enseñar matemática y disposición para hacer bien la tarea (Díaz y Poblete, 2009a, 2009b, 2016b).

Con base en estos referentes teóricos, se toman decisiones para realizar el rediseño curricular y escoger el enfoque pedagógico y las herramientas metodológicas que lo acompañarán. La concreción de esta innovación curricular, consideró la reflexión analítica con respecto al concepto de competencias. A partir de esta discusión se elaboró el perfil profesional y los respectivos cursos, tarea que debió evidenciar la coherencia entre las competencias, los módulos de aprendizaje y el perfil profesional. Esta orientación curricular promueve la vivencia de la relación dialéctica entre teoría y práctica, aspecto fundamental para que el futuro profesional graduado se vincule al campo laboral.

\section{Metodología}

Las estrategias y actividades programadas para el desarrollo del rediseño curricular, se iniciaron en
2010 y se dividieron en cinco etapas de trabajo previas a su implementación en la carrera de Pedagogía en Matemática y Computación, como se muestra en la figura 2.

\section{Etapa 1: perfeccionamiento docente}

Se realizaron dos tipos de perfeccionamiento:

- In situ, teniendo como marco conceptual el desarrollo de un currículo por competencias con asistencia de la totalidad de los académicos de la carrera a talleres de competencias didáctico-matemático, didáctico-computacional y didáctico-pedagógico, dictados por expertos de universidades nacionales e internacionales, especialistas en estrategias didácticas y en evaluación de aprendizajes en competencias. Las capacitaciones fueron conducentes a diplomados y especializaciones atingentes al enfoque por competencias, además de asistencia de los académicos a congresos sobre el tema.

- Estadías de académicos, realizadas en centros de educación superior de Europa y América, con especialización en adaptación de currículo por competencias, así como en didácticas especiales concernientes a las líneas de formación disciplinarias en matemática y computación.

\section{Figura 2. Etapas del Rediseño Curricular}

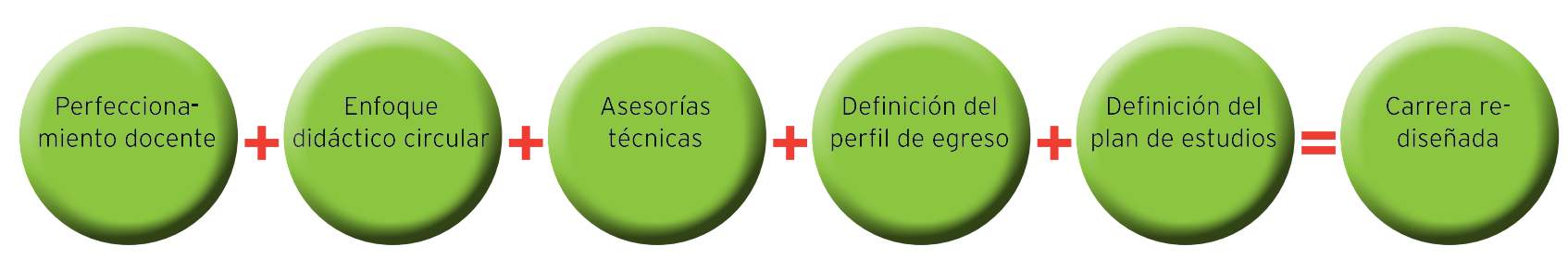

Fuente: elaboración propia con base en el proyecto MECESUP ULA0608. 


\section{Etapa 2: enfoque didáctico-curricular}

En esta etapa se definieron las competencias disciplinarias, competencias didáctico-pedagógicas y competencias para la formación general, para integrar y contextualizar el saber y saber-hacer, ser y saber-ser, mediante indicadores de desempeño agrupados en tres niveles de desarrollo de las competencias (nivel 1 , nivel 2 y nivel 3).

\section{Competencias disciplinarias}

- Conocimiento del contenido matemático, informático y de computación, su valor formativo y cultural, su historia, epistemología y aplicaciones.

- Habilidad para la resolución de problemas matemáticos, informáticos y de computación; la comunicación de ideas matemáticas, informáticas y de computación, y la conexión entre diferentes contextos.

La tabla 1 integra los tres niveles de desarrollo de las competencias disciplinarias.

\section{Competencias didáctico-pedagógicas}

- Conocimiento del desarrollo psicosocial del alumnado y de la didáctica de la matemática y utilizarlo como uno de los fundamentos del proceso de enseñanza aprendizaje de la matemática en educación secundaria, que permita enfrentar la diversidad del alumnado.

- Capacidad para propiciar un ambiente favorable para el aprendizaje de la matemática y para planificar y programar la intervención educativa de acuerdo con las teorías de aprendizaje de la matemática, mediante diferentes estrategias de enseñanza según el contexto.

- Capacidad para aplicar formas e instrumentos de evaluación actualizados y adaptados a las características de la matemática, a los objetivos fundamentales y transversales, y a las competencias matemáticas a alcanzar.
- Capacidad para analizar procesos de enseñanza y aprendizaje en matemática, para aplicar criterios de calidad y para generar proyectos de mejoramiento de la enseñanza de la matemática, con énfasis en su propia práctica.

\section{Competencias generales}

- Capacidad para comunicarse correctamente en forma oral y escrita con un discurso racional, utilizando adecuadamente las TIC e incorporando el uso del inglés como herramienta para el desarrollo profesional.

\section{Etapa 3: asesorías técnicas}

Se diseñó un plan específico de asesoría externa, que implicó la contratación de personal técnico que elaboró y aplicó, en primera instancia, la evaluación diagnóstica de competencias básicas en los estudiantes que ingresaron a la carrera. Del mismo modo, los expertos realizaron entrevistas fenomenológicas a los académicos, focalizadas por áreas temáticas de los programas de estudios de las líneas de matemática y computación. También realizaron la planificación de relaciones de competencias en el plan de estudios, malla y programa para diseñar procedimientos evaluativos por cada año y acumulados hasta el cuarto año de la carrera. Finalmente, los expertos elaboraron instrumentos evaluativos de consulta a egresados de la carrera, a alumnos de los egresados y a los empleadores, lográndose estimar cinco variables: dominio de la especialidad, metodologías, responsabilidad, relación de la matemática con otras disciplinas, uso de medios y TIC. Posteriormente, para lograr experticia en estrategias de enseñanza para el desarrollo de competencias, se requirió personal especializado en orientaciones didácticas específicas, evaluación de los aprendizajes y técnicas estadísticas e informáticas, lo cual se sistematizó en la etapa 4 que se describe a continuación. 
Tabla 1. Niveles de competencias disciplinarias

\begin{tabular}{|l|l|l|}
\hline \multicolumn{1}{|c|}{ Nivel 1 } & \multicolumn{1}{c|}{ Nivel 2 } & \multicolumn{1}{c|}{ Nivel $\mathbf{3}$} \\
\hline $\begin{array}{l}\text { Conocimientos de los contenidos } \\
\text { matemáticos del currículum de la } \\
\text { formación general y diferenciada de la } \\
\text { educación secundaria. }\end{array}$ & $\begin{array}{l}\text { Capacidad para relacionar su } \\
\text { conocimiento matemático, informático } \\
\text { y de computación adquirido con los } \\
\text { contenidos del currículum de educación } \\
\text { secundaria. }\end{array}$ & $\begin{array}{l}\text { Capacidad para profundizar y ampliar los } \\
\text { contenidos del currículum obligatorio de } \\
\text { pedagogía en matemática y computación. }\end{array}$ \\
\hline
\end{tabular}

Fuente: elaboración propia con base en el proyecto MECESUP ULA0608.

Tabla 2. Niveles de competencias didáctico-pedagógicas

\begin{tabular}{|l|l|l|}
\hline \multicolumn{1}{|c|}{ Nivel 1 } & \multicolumn{1}{|c|}{ Nivel $\mathbf{2}$} & \multicolumn{1}{c|}{ Nivel $\mathbf{3}$} \\
\hline $\begin{array}{l}\text { Conocimiento de los contenidos } \\
\text { psicológicos, sociales, pedagógicos y } \\
\text { didácticos necesarios para la enseñanza } \\
\text { de la matemática del currículum de la } \\
\text { educación secundaria. }\end{array}$ & $\begin{array}{l}\text { Capacidad para relacionar sus } \\
\text { conocimientos psicológicos, sociales, } \\
\text { pedagógicos y didácticos adquiridos, } \\
\text { con los contenidos del currículum de la } \\
\text { educación secundaria. }\end{array}$ & $\begin{array}{l}\text { Capacidad para aplicar y profundizar sus } \\
\text { conocimientos psicológicos, sociales, } \\
\text { pedagógicos y didácticos. }\end{array}$ \\
\hline
\end{tabular}

Fuente: elaboración propia con base en el proyecto MECESUP ULA0608.

Tabla 3. Niveles de competencias para la formación general

\begin{tabular}{|l|l|l|}
\hline \multicolumn{1}{|c|}{ Nivel 1 } & \multicolumn{1}{|c|}{ Nivel $\mathbf{2}$} & \multicolumn{1}{c|}{ Nivel $\mathbf{3}$} \\
\hline $\begin{array}{l}\text { Capacidad para comprender diversos } \\
\text { lenguajes (lengua materna, inglés, TIC) } \\
\text { en diferentes contextos. }\end{array}$ & $\begin{array}{l}\text { Capacidad para comprender diversos } \\
\text { lenguajes (lengua materna, inglés, TIC) } \\
\text { en contextos profesionales. }\end{array}$ & $\begin{array}{l}\text { Capacidad para comprender diversos } \\
\text { lenguajes (lengua materna, inglés, TIC) } \\
\text { en espacios de participación profesional } \\
\text { específicos (revistas, congresos, etcétera). }\end{array}$ \\
\hline
\end{tabular}

Fuente: elaboración propia con base en el proyecto MECESUP ULA0608.

\section{Etapa 4: definición del perfil de egreso}

Se elaboró una propuesta de perfil profesional siguiendo el Modelo Educativo Institucional de la Universidad y tomando como referencia el enfoque didáctico-curricular detallado en la etapa 1 . Se consideraron los estándares pedagógicos y disciplinares del Ministerio de Educación, el Marco para la Buena Enseñanza y los planes y programas de estudio de la enseñanza secundaria, quedando finalmente definido el perfil de egreso como "Un titulado con actitudes, habilidades y conocimientos de la matemática e informática, principalmente para su enseñanza en la educación secundaria, con formación educativa centrada en el desarrollo de valores, que integre los contenidos matemáticos e informáticos superiores y de enseñanza secundaria en contextos de la vida cotidiana, vinculando su enseñanza con procesos didácticos y de investigación".

Finalizadas las cuatro etapas de trabajo, se generó un diseño que fue socializado ampliamente, hasta concluir a fines de 2010 con un nuevo plan de estudios y puesto en práctica en una primera cohorte en marzo de 2011.

\section{Etapa 5: definición del plan de estudios}

En esta etapa se organizaron las competencias del perfil de egreso y los resultados de aprendizaje, para 
el diseño de los programas de asignaturas y asignación de créditos. Se estructuró el plan de estudio de la carrera en torno a cuatro líneas de formación: formación de especialidad, formación pedagógica, formación general (competencias generales y transversales), y prácticas profesionales. En cada una de las líneas se diseñaron nuevas asignaturas o se actualizaron los contenidos de las ya existentes, respondiendo a las competencias del perfil de egreso y a los resultados de aprendizaje.

Las principales innovaciones se generaron en la línea de formación disciplinaria al agregar al plan de estudio la asignatura de Matemática Básica (primer semestre); en la línea de formación pedagógica, al incluir Metodología de la Investigación y Seminario de Grado, permitiendo con éste último separar el grado académico del título profesional; en la formación general se aumentó significativamente el idioma inglés, pudiendo los estudiantes optar a la certificación internacional TOEIC, además de la implementación de cursos referidos a competencias generales y competencias transversales, que contribuyen a su desarrollo desde una visión contextual ética y pluralista.

\section{Resultados}

Los resultados que se presentan en este artículo provienen del proceso de autoevaluación al que se sometió la carrera, luego de la implementación de cinco años de una renovación curricular por competencias. Por tal razón, se explicitan en función de las tres dimensiones exigidas para el aseguramiento de la calidad en carreras de pregrado en las universidades en Chile. También se incluyen los resultados obtenidos en las tasas de egreso y titulación. Cabe hacer notar que el desarrollo propio de cada etapa presentada también contiene resultados que surgieron como producto del proceso concreto de rediseño curricular.

\section{Tasas de titulación}

Se verificó un mejoramiento sustantivo en las tasas de egreso y titulación en el periodo 2011-2015, que corresponde a la cohorte con rediseño curricular por competencias. La tabla 4 contiene la evolución de la matrícula de la carrera y su titulación.

A través del resumen presentado en la tabla 4, se aprecia un notorio incremento en el total de los egresos y titulaciones en la carrera. El rediseño curricular ha contribuido significativamente a disminuir la lenta progresión de los estudiantes. Si se analiza la información de la primera generación con nueva malla curricular, las cifras cambian notoriamente, situación que históricamente no ocurría con cohortes anteriores.

En 2016 la carrera se sometió a un proceso de autoevaluación con miras a certificar calidad asociada a su rediseño curricular en competencias, lo que de acuerdo con la nueva exigencia legal, la realiza sólo la GNA y basada en la evaluación de la calidad de la formación ofrecida, se operacionaliza en tres dimensiones: Perfil de Egreso y sus Resultados, Condiciones de Operación y Capacidad de Autorregulación,

Tabla 4. Evolución de la matrícula y titulación en la carrera

\begin{tabular}{|l|c|c|c|c|c|}
\hline & $\mathbf{2 0 1 1}$ & $\mathbf{2 0 1 2}$ & $\mathbf{2 0 1 3}$ & $\mathbf{2 0 1 4}$ & $\mathbf{2 0 1 5}$ \\
\hline Matriculados $1^{\circ}$ año & 24 & 13 & 19 & 16 & 17 \\
\hline Matrícula Total & 180 & 164 & 156 & 146 & 134 \\
\hline Titulados & 13 & 14 & 17 & 23 & 32 \\
\hline
\end{tabular}

Fuente: Informe de autoevaluación de Pedagogía en Matemáticas y Computación, 2016. 
las cuales contemplan a su vez criterios de calidad que definen los parámetros que tiene que cumplir la carrera de Pedagogía en Matemática y Computación para acreditar que realiza su trabajo con calidad.

El proceso de autoevaluación requirió de la aplicación de encuestas de satisfacción online, de carácter voluntario y emanadas de la CNA, a un total de 164 personas. En relación con la dimensión Perfil de Egreso y sus Resultados, ésta aborda los diferentes aspectos que atañen a la carrera y que determinan su pertinencia y calidad. En particular, en esta sección se dan a conocer aspectos centrales del programa académico, como son el perfil de egreso y la estructura curricular de la carrera, la efectividad del proceso formativo y los resultados más importantes alcanzados a través de su implementación. La dimensión Condiciones de Operación aborda temáticas referentes a la estructura organizacional, administrativa y financiera bajo la cual se desenvuelve el programa de formación, como también los recursos humanos, de infraestructura y técnicos con que cuenta para el cumplimiento de sus objetivos. En último término se analiza la dimensión Capacidad de
Autorregulación que demuestra tener la carrera. El análisis se centra en los objetivos educacionales declarados por la carrera y en la coherencia existente entre éstos y los objetivos institucionales y los de la Escuela de Pedagogía, de la que depende administrativamente, y el Departamento de Ciencias Exactas, del que depende disciplinariamente.

\section{Resultados por criterio de calidad de los estudiantes}

En el caso de los estudiantes, la muestra lograda fue de 78 estudiantes de la carrera, que corresponde al $73 \%$ de la matrícula total de 2016. Al ser consultados, se alcanzaron como promedio general en cada uno de los diez criterios, los porcentajes de satisfacción que se detallan en el gráfico 1.

Los estudiantes concitan un consenso de $81.8 \%$ de satisfacción en la totalidad de los criterios. Comparativamente, todas las dimensiones de esta autoevaluación superan las logradas en el proceso de autoevaluación anterior (2006-2011), en la que en promedio se alcanzó en las mismas dimensiones sólo un $62.2 \%$ de satisfacción de las cohortes encuestadas.

\section{Gráfico 1. Resultados promedio general por criterio de los estudiantes}

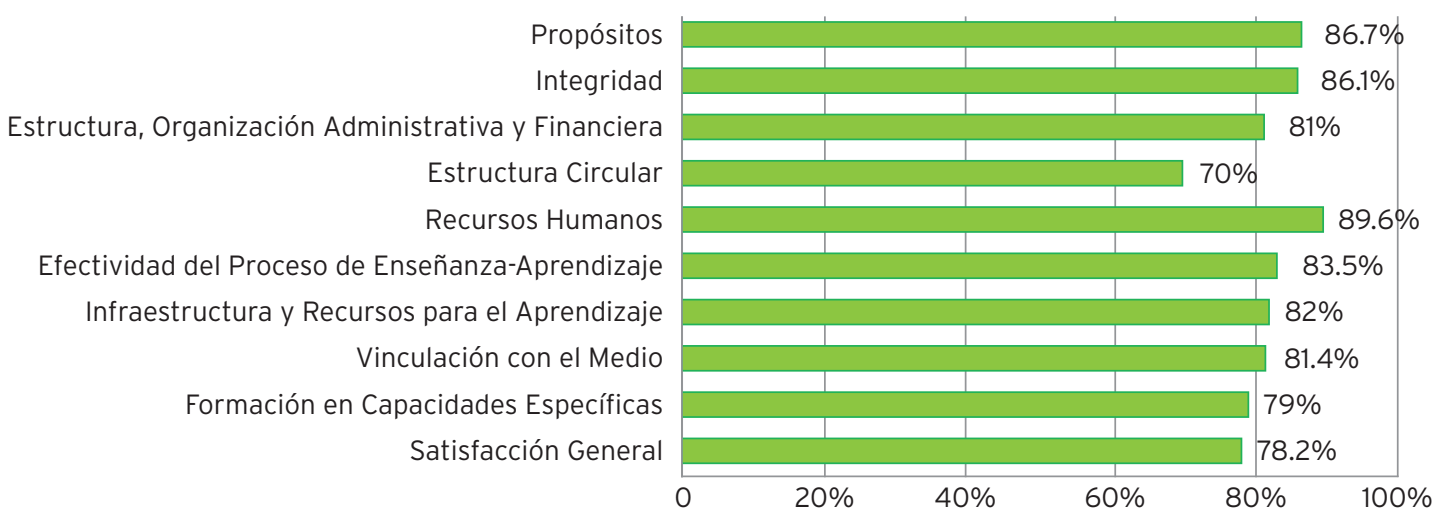

Fuente: Informe de autoevaluación de Pedagogía en Matemáticas y Computación, 2016. 


\section{Resultados por criterio de calidad de los académicos}

En lo que respecta a los académicos, la encuesta la respondieron un $79 \%$ del total de docentes que prestan servicio a la carrera y que, por tanto, se vinculan al proceso de formación de la misma. Los porcentajes promedios de satisfacción de los académicos en cada uno de los once criterios consultados se muestran en el gráfico 2.

En promedio, la aprobación de los académicos en la totalidad de los criterios es muy alta y alcanza el $90.5 \%$ de satisfacción. En la autoevaluación anterior (2006-2011) se registró un 90.4\% de aprobación en los mismos criterios.

\section{Resultados por criterio de calidad de los empleadores}

Con respecto a los empleadores, el cuestionario fue respondido por 15 , lo que representa un $88 \%$ de un total de 17 a quienes se les hizo llegar la encuesta (4 más que en el proceso anterior). De ellos, el 66.7\% pertenecen al sector particular subvencionado; del resto, $26.7 \%$ son profesionales vinculados al sector público y el $6.7 \%$ pertenecen al sector de educación privada. En cuanto al tamaño y tipo de organización de la que proviene el grupo de informantes empleadores, en su mayoría se trata de organizaciones educacionales medianas $(60 \%)$ que tienen entre 31 y 99 funcionarios, en menor grado a organizaciones educativas de gran tamaño $(26.7 \%$ de los empleadores), en tanto que sólo un $13.3 \%$ de la muestra estuvo constituido por profesionales empleadores de instituciones pequeñas, manteniéndose la tendencia presentada en el proceso de evaluación anterior. Los porcentajes promedios de satisfacción de los empleadores, en cada uno de los siete criterios consultados, se muestran en el gráfico 3 .

\section{Resultados por criterio de calidad de los egresados}

La encuesta se aplicó al 37.2\% del total de egresados/titulados por cohorte de ingreso a la carrera entre los años 2002-2011. Los porcentajes promedios de satisfacción de los egresados de la carrera, en cada uno de los once criterios consultados, se muestran en el gráfico 4.

En promedio, la aprobación de los egresados en la totalidad de los criterios es muy alta y alcanza $85.2 \%$ de satisfacción. Comparativamente y al igual que los estudiantes, los egresados encuestados en este proceso, superan el grado de aprobación de los criterios de la autoevaluación anterior (2006-2011), en la que lograron un $76.7 \%$ de aprobación.

\section{Gráfico 2. Resultados promedio general por criterio de los académicos}

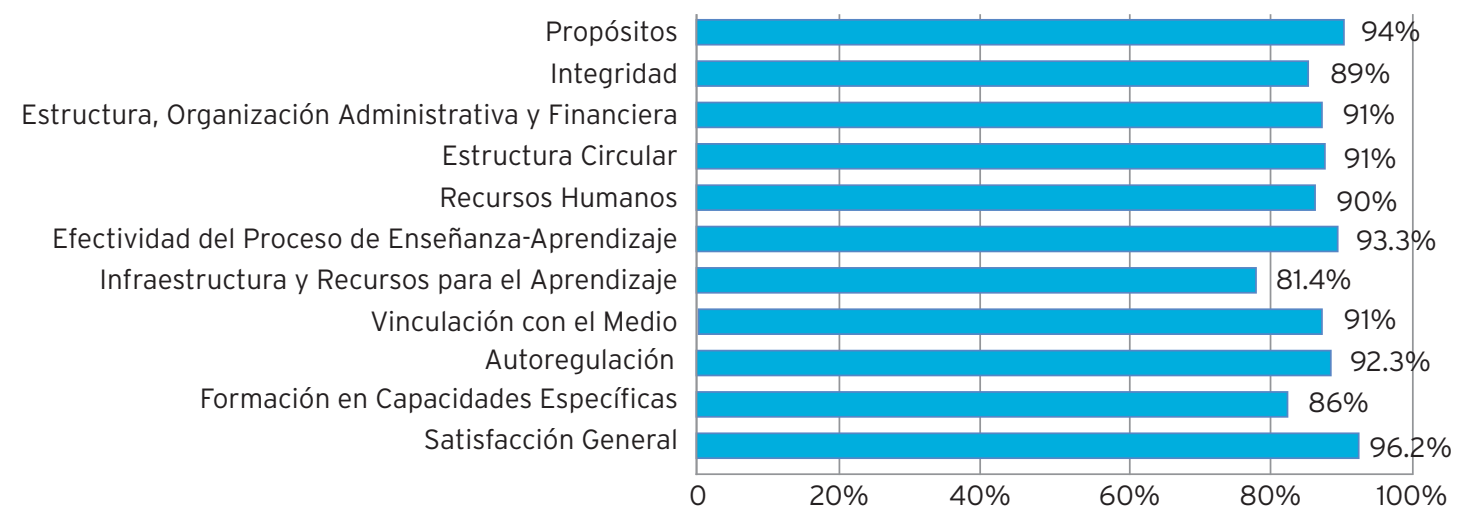

Fuente: Informe de autoevaluación de Pedagogía en Matemáticas y Computación, 2016. 


\section{Gráfico 3. Resultados promedio general por criterio de los empleadores}

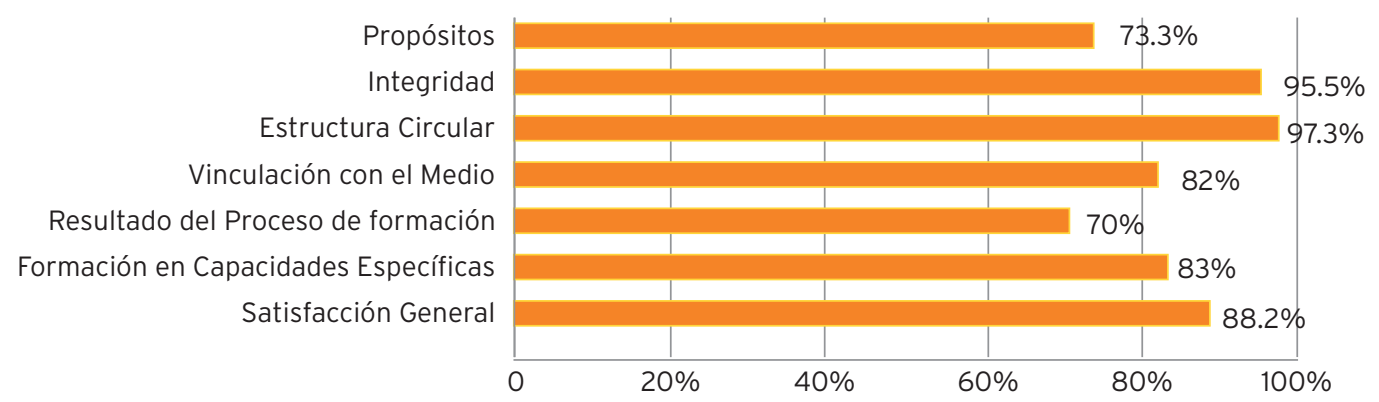

Fuente: Informe de autoevaluación de Pedagogía en Matemáticas y Computación, 2016.

\section{Gráfico 4. Resultados promedio general por criterio de los egresados}

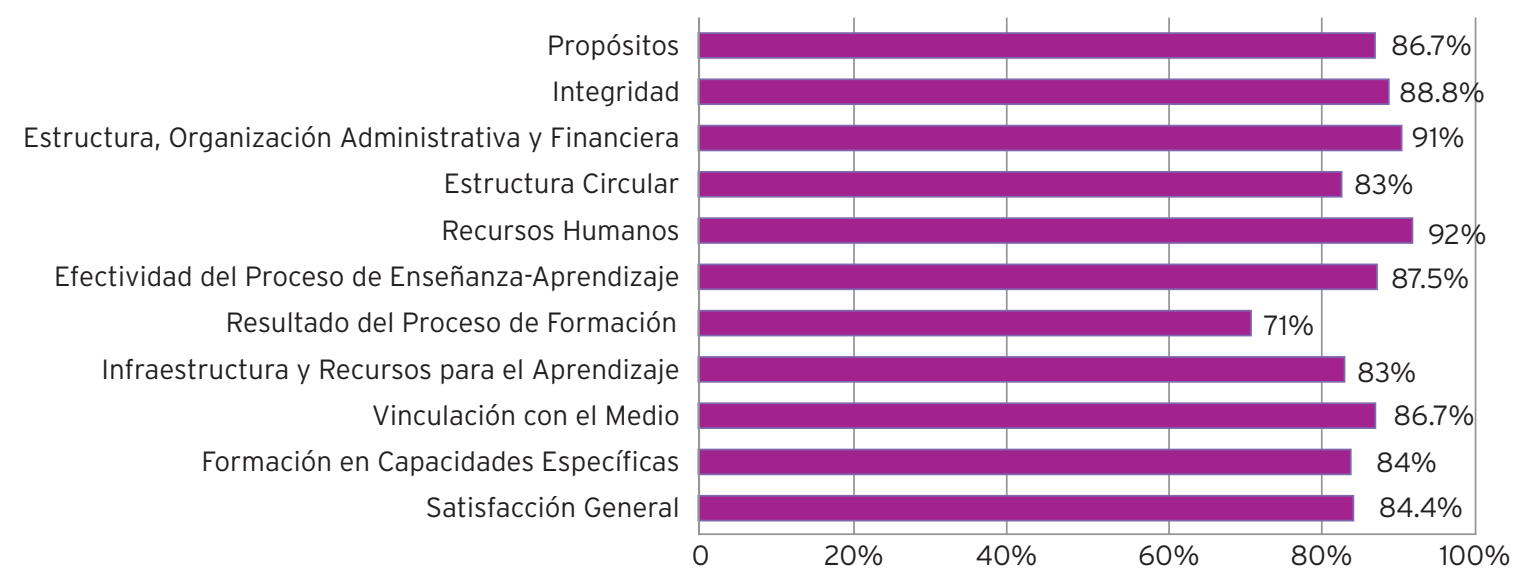

Fuente: Informe de autoevaluación de Pedagogía en Matemáticas y Computación, 2016.

\section{Resultados por dimensión}

En la dimensión Perfil de Egreso y Resultados, las fortalezas encontradas en el proceso de autoevaluación indican que el perfil es conocido y valorado por todos los actores que formaron parte del proceso, además de reconocido y evaluado por asesores externos a la institución. Por su parte, el plan de estudios prepara para el desempeño laboral y se caracteriza por una fuerte consistencia entre las líneas de formación disciplinaria-pedagógica y perfil de egreso. También tiene pertinencia la formación ofrecida en competencias, con las exigencias del mundo profesional. Sin embargo, las debilidades que se le reconocen al plan de estudios, son la existencia de materias poco significativas en la formación y escaso fomento de la creatividad en algunas asignaturas.

En la subdimensión resultados del proceso de formación, se valora la pertinencia de la formación, en términos del despliegue de competencias demostrado por los titulados en el campo laboral, el importante aumento en la tasa de titulación; sin embargo, se reconoce una insuficiente política de colocación laboral.

En la dimensión Condiciones de Operación, las fortalezas encontradas en el proceso, indican que se destaca la implementación de medios tecnológicos que ocupa la carrera y sistemas bibliográficos. En lo relativo al recurso humano, los docentes, además de 
suficientes en cantidad, son de alta excelencia y vistos como idóneos para la función que desempeñan y con conocimientos actualizados en su disciplina, con experticias en los diversos ámbitos de desarrollo, dado que mayoritariamente son magister y doctores.

En la subdimensión Estructura Organizacional, Administrativa y Financiera, constituyen fortalezas la administración y funcionamiento del Departamento de Ciencias Exactas que tutela disciplinariamente a la carrera, además de la gestión de recursos que se reconoce como ordenada y coordinada con las distintas instancias administrativas de la Universidad. Pero se reconoce como debilidad la burocracia para realizar trámites administrativos de los estudiantes.

En la dimensión Capacidad de Autorregulación, las fortalezas encontradas en el proceso de autoevaluación, en lo referido a los propósitos, indican que existe una clara y formal definición de la misión y propósitos de la carrera, en articulación con los de la Universidad, con un ambiente académico que se caracteriza por su continua reflexividad y capacidad crítica. Sin embargo, la debilidad de la dimensión la formulan los empleadores al señalar que las autoridades de la carrera no consultan regularmente sus opiniones.

Finalmente, en la subdimensión Integridad, se destacan las normativas y reglamentaciones formales, las decisiones de los cuerpos directivos de la carrera tomadas con criterios académicos, y la valoración positiva de la formación y su relación con el cumplimiento de los objetivos de la carrera.

\section{Conclusiones y discusión}

Realizar transformaciones en la formación de profesores, implica asumir importantes desafíos en las instituciones de educación superior. En este contexto de cambio, damos cuenta de los resultados de un proyecto de investigación y desarrollo que, en cinco años de ejecución, reformuló el diseño de una carrera logrando la integración y la contextualización de formas didácticas y de contenido matemático, informático y de computación, con la implementación de un currículo por competencias, en la formación de estudiantes de Pedagogía en Matemática y Computación en la Universidad de Los Lagos de Osorno en Chile.

Los primeros resultados surgieron del proceso de autoevaluación de la carrera y su certificación de calidad por la GNA, que en la actualidad cuenta con un nuevo plan de estudio, un perfil de egreso adecuado al currículo de formación basado en competencias e importante mejoramiento en las tasas de aprobación, retención y titulación, además de fortalecimiento de habilidades fundamentales de los estudiantes.

Con el rediseño curricular, tanto el perfil de egreso y la estructura curricular de la carrera han sido claramente definidos. El perfil de egreso fue elaborado considerando el estadio de desarrollo de los fundamentos científicos, disciplinarios y pedagógicos propios de una carrera de formación de profesores en las áreas de la matemática y de la computación. El plan de estudios y la estructura curricular por competencias integran en forma equilibrada líneas de formación en matemática, computación e informática y educación. Se caracteriza por propender a una adecuada vinculación entre los conocimientos teóricos y prácticos, a través del desarrollo de una diversidad de trabajos que son requeridos por las líneas de formación y asignaturas de dicho plan asociados a un sistema de crédito transferible.

Los logros, en general, están íntimamente relacionados con el efectivo desarrollo de las siguientes cinco etapas de trabajo propuestas en este proyecto de investigación, que permitió el rediseño curricular: perfeccionamiento docente; enfoque didáctico-curricular, que comprende la elaboración y validación técnica de matrices de competencias por líneas de especialidad, que permitieron integrar y contextualizar el saber, saber-hacer, ser y saber-ser; didácticoespecífico y tecnológico con base en la utilización de un específico Modelo de Competencias Profesionales 
de Matemática (MCPM) (Poblete y Díaz, 2004); asistencia técnica; definición del perfil de egreso, y definición del plan de estudio. Todo lo expuesto, refleja la alta capacidad que tiene la carrera de autorregulación, así como también de responder oportunamente a las políticas nacionales, asociada a la calidad en Chile.

La implementación del rediseño curricular basado en competencias muestra que los primeros resultados concretos son aquellos que surgieron del proceso mismo de la renovación curricular, es decir, un nuevo plan de estudio formulado en forma técnica y especializada, de forma sistemática y participativa. La definición de un perfil de egreso con competencias pertinentes y medibles, que se traducen en un itinerario formativo a través de la malla curricular, que a su vez se plasma en los programas de cada curso en que se pensó y planificó la metodología necesaria para que se puedan alcanzar y evaluar los resultados de aprendizaje.

De acuerdo con Beneitone et al. (2007), uno de los problemas más comunes en los rediseños curriculares que se orientan al logro de competencias, es lo que el autor denomina el "divorcio" que se establece entre las competencias que se formulan en el perfil de egreso y las propuestas de enseñanza de los profesores en cada asignatura.

$\mathrm{Al}$ respecto debemos señalar que fue indispensable capacitar a los profesores para la preparación y el manejo de la metodología, ya que para llevar a la práctica un rediseño del currículo, lo primero es contar con docentes que manejan la metodología, que viven la cultura del modelo educativo. Ello requiere que estén capacitados, no sólo en el manejo de métodos sino también en la filosofía educativa que los sustenta. De esta forma, se generó una cultura común que es un factor central para que el modelo educativo sea sostenible en el tiempo. Por su parte, los estudiantes respondieron muy bien a la metodología, fueron adaptándose a ella progresivamente y encontrando su valor.
A la luz de los resultados obtenidos, estamos ciertos que la utilización de este enfoque por competencias permitió expresar mejor las capacidades que tienen los titulados al momento de completar sus estudios en la carrera, lo cual facilita el proceso de transición que ocurre entre el término de los estudios en la Universidad y la incorporación al ejercicio laboral. Sin embargo, en el proceso, hubo muchos aprendizajes, dudas y dificultades que vencer y decisiones que tomar, que se convierten en lecciones aprendidas que es necesario compartir.

La primera reflexión se refiere al significado del currículo y sus implicancias. Es común pensar que un currículo es un listado ordenado de asignaturas, que se distribuyen en forma secuencial en el tiempo. Nos parece que un currículo es mucho más que eso: incluye definir los resultados que se espera alcanzar, los contenidos, las competencias, las estrategias, la metodología, la evaluación y los recursos a emplear. Es un proceso de construcción de aprendizajes que articula todos los componentes para el logro de los resultados, que responden a las necesidades académicas.

Otro tema que requiere discusión es el concepto de competencia. Algunos describen una competencia como un listado de conocimientos, habilidades y actitudes que se dan simultáneamente y que el alumno debe memorizar. Una competencia es mucho más que eso, es un aprendizaje complejo que requiere la integración de estos tres elementos en forma integral, de modo que se conviertan en un comportamiento habitual. Esto sólo se logra cuando el estudiante participa en forma activa en experiencias de aprendizaje adecuadas, de manera que su manera de pensar y de actuar cambia, ello se refleja en conductas observables y medibles.

El perfil profesional para algunos es una especie de declaración de principios que no necesariamente guarda relación con la actividad educativa. Por el contrario, en el proceso descrito, el perfil fue la base del proceso de diseño, las especificaciones para 
construir el mapa curricular y para definir los contenidos, las estrategias, la metodología y los recursos. El perfil vincula el currículo a la realidad del contexto en que se desarrolla, para mantener esta vinculación es necesario hacer un esfuerzo continuo

\section{Referencias}

Álvarez, Margarita (2011), "Perfil del docente en el enfoque basado en competencias", en Revista Electrónica Educare, vol. 15, núm. 1, pp. 99-107.

Arnaz, José (1993), La planeación curricular, $2^{a}$ edición, México, Trillas.

Ávalos, Beatrice (2014), "La formación inicial docente en Chile: tensiones entre políticas de apoyo y control", en Estudios Pedagógicos, vol. XL, núm. 1, pp. 11-28.

Ávalos, Beatrice y Claudia Matus (2010), La formación inicial docente en Chile desde una óptica internacional. Informe nacional del estudio internacional IEA TEDS-M, Santiago de Chile, Ministerio de Educación.

Avendaño, Catherine, Karol Gutiérrez, Camila Salgado y Manuel Dos-Santos (2016), "Rendimiento académico en estudiantes de Ingeniería Comercial: modelo por competencias y factores de influencia”, en Formación Universitaria, vol. 9, núm. 3, pp. 3-10 [DOI: 10.4067/ S0718-50062016000300002].

Bell, Allison y Kristin Conklin (2013), State financial aid programs and competency-based education, Washington, HCM Strategists.

Beneitone, Pablo, César Esquetini, Julia González, Maida Marty Maletá, Gabriela Siufi y Robert Wagenaar (2007), Reflexiones y perspectivas de la educación superior en América Latina. Informe final. Proyecto Tuning América Latina 2004-2007, Bilbao, Universidad de Deusto.

Bolívar-Cruz, Alicia, Nancy Dávila-Cárdenes, Sara González-Betancor, Alexis López-Puig, Heriberto Suárez-Falcón y Domingo Verano-Tacoronte (2011), "Las competencias transversales y el Espacio Europeo de Educación Superior (EEES): una experiencia de de análisis y ajuste. Ello requiere liderazgo de las autoridades, participación activa de los académicos y una organización adecuada que soporte el cambio, de lo contrario la entropía se encargará de destruir lo avanzado. evaluación de la competencia de comunicación oral en la ULPGC", en Jornadas Internacionales de Innovación Universitaria, 8, Actas de Congreso, Madrid, Universidad Europea de Madrid.

Boyatzis, Richard Eleftherios (2007), "Competencies in the 21 st century", en Fournal of Management Development, vol. 27, núm. 1, pp. 5-12.

Burger, Edward y Michael Starbird (2005), The heart of mathematics: an invitation to effective thinking (2a ed.), Emeryville, EUA, Key College Publishing.

Bustamante, Miguel A., Claudio Oyarzún, Maderline Grandón y Caroline Abarza (2015), "Fundamentos de la enseñanza por competencias a nivel de postgrado en dos universidades públicas chilenas", en Formación Universitaria, vol. 8, núm. 6, pp.23-30.

Cisterna, Cecilia, Valentina Soto y Constanza Rojas (2016), "Rediseño curricular en la Universidad de Concepción: la experiencia de las carreras de formación inicial docente", en Calidad en la Educación, vol. 44, pp. 301-323.

Cox, Cristian, Lorena Meckes y Martin Bascopé (2010), "La institucionalidad formadora de profesores en Chile en la década del 2000: velocidad del mercado y parsimonia de las políticas", en Pensamiento Educativo, vol. 46-47, pp. 205-245.

Consejo de Rectores de las Universidades Chilenas (GRUCH) (2012), Innovación curricular en las universidades del consejo de rectores, $1^{\text {a }}$ edición, Chile, Nuevamérica Impresiones, pp. 1-190.

Cuba, Amadeo (2016), "Constructo competencia: síntesis histórico-epistemológica", en Educación, vol. XXV, 
núm. 48, pp. 7-27.

Daugherty, Lindsay, Van Davis y Trey Miller (2015), Competency-based education programs in Texas: an innovative approach to higher education, Santa Monica, California, RAND Corporation.

Díaz, Verónica y Álvaro Poblete (2016a), "A model of professional competences in mathematics and didactic knowledge of teachers", en International fournal of Mathematical Education in Science and Technology, pp. 1-13 [DOI: 10.1080/00207].

Díaz, Verónica y Álvaro Poblete (2016b), "Modelo de competencias profesionales de matemáticas y su implementación en profesores de la enseñanza primaria", en Mathematics Education Bulletin, vol. 30, núm. 55,pp. 786-807 [DOI: 10.1590/1980-4415v30n55a23].

Díaz, Verónica y Alvaro Poblete (2015), "Training in mathematics based on competences: successful experiences in Chilean schools", paper in proceedings, 8th International Conference of Education, Research and Innovation, Sevilla, España, pp. 3059-3067, ICERI (eds.), IATED Academy.

Díaz, Verónica y Alvaro Poblete (2009a), "Competencias y transposición didáctica: binomio para un efectivo perfeccionamiento en matemática", en Revista de Investigación y Postgrado, vol. 24, núm. 2, pp. 77-107.

Díaz, Verónicay Alvaro Poblete(2009b), "Perfeccionamiento en matemática basado en competencias para docentes de escuelas básicas municipalizadas de la Región de Los Lagos y de Los Ríos", en Revista Estudios Pedagógicos, vol. 35, núm. 2, pp. 13-34.

Dochy, Filip y Goele Nickmans (2005), Competentiegericht opleiden en toetsen: theorie en praktijk van flexibel lerén, Lemma, Utrecht.

Fletcher, Shirley (1997), Analysing competence: Tools and techniques for analyzing jobs, roles and functions, Londres, Kogan Page.

Guzmán, María A., Oscar Maureira, Alejandra Sánchez y Adriana Vergara (2015), "Innovación curricular en la educación superior: ¿cómo se gestionan las políticas de innovación en los (re) diseños de las carreras de pregrado en Chile?", en Perfiles Educativos, vol. 37, núm.
149, pp. 60-73.

Hambur, Sam, Ken Rowe y Le T. Luc (2002), Graduate skills assessment: stage one validity study, Canmberra, Department of Education, Science and Training.

Hill, Heather, Merrie Blunk, Charalambo Charambous, Jennifer Lewis, Geoffrey Phelps, Laurie Sleep y Deborah L. Ball (2008), "Mathematical knowledge for teaching and the mathematical quality of instruction. An exploratory study", en Cognition and Instruction, vol. 26, núm. 4, pp. 430-511 [DOI: 10.1080/07370000802177235].

Icarte, Gabriel y Hugo Labate (2016), "Metodología para la revisión y actualización de un diseño curricular de una carrera universitaria incorporando conceptos de aprendizaje basado en competencias", en Formación Universitaria, vol. 9, núm. 2, pp. 3-16 [DOI: 10.4067/ S0718-50062016000200002].

Informe de Autoevaluación de Pedagogía en Matemáticas y Computación (2006), Osorno, Universidad de los Lagos.

Kelchen, Robert (2015), The landscape of competencybased education: enrollments, demographics, and affordability, Washington, American Enterprise Institute (AEI Series on Competency-Based Higher Education).

Le Boterf, Guy (2001), Ingeniería de las competencias, Barcelona, Gestión 2000.

López-Ruiz, Juan Ignacio (201 1), "Un giro copernicano en la enseñanza universitaria: formación por competencias", en Revista de Educación, vol. 356, pp. 279-301.

Lozano, José Félix, Alejandra Boni, Jordi Peris y Andrés Hueso (2012), "Competencies in higher education: a critical analysis from the capabilities approach", en Fournal of Philosophy of Education, vol. 46, núm. 1, 132147 [DOI: 10.1111/j.1467-9752.2011.00839.x].

Mansfield, Richard S. (1996), "Building competency models: approaches for HR professionals", en Human Resource Management, vol. 35, núm. 1, pp. 7-18 [DOI: 10.1002/(SICI) 1099-050X(199621)35:1<7::AIDHRM1>3.0.CO;2-2].

Markus, Leanne H., Helena D. Cooper-Thomas y Keith N. Allpress (1997), "Confounded by competencies? An evaluation of the evolution and use of competency 
models", en New Zealand Fournal of Psychology, vol. 34, núm. 2, pp. 117-126.

Meckes, Lorena y Martín Bascopé (2012), "Uneven distribution of novice teachers in the Chilean Primary School System", en Education Policy Analysis Archives, vol. 20, núm. 30, <http://epaa.asu.edu/ojs/article/ view/1017>.

Muñoz-Osuna, Francisca, Antonio Medina-Rivilla y Manuela Guillén-Lúgigo (2016), "Jerarquización de competencias genéricas basadas en las percepciones de docentes universitarios", en Educación Química, vol. 27, pp. 126-132 [DOI: 10.1016/j.eq.2015.11.002].

Nassif, Jorge, Mori Hanashiro y Rosane Torres (2010), "Fatores que influenciam na percepção das competências para o exercício da docência”, en Revista Brasileira de Educação, vol. 15, núm. 44, pp. 364-412.

Nodine, Thad (2016), "How did we get here? A brief history of competency-based higher education in the United States", en Competency-based Education, vol. 1, pp. 5-11 [DOI: 10.1002/cbe2.1004].

OCDE (2013), El aseguramiento de la calidad en la educación superior en Chile, OECD Publishing [DOI: 10.1787/9789264191693-es].

OCDE (2012), Testing student and university performance globally: OECD's AHELO, <www.oecd.org/edu/ahelo>.

OCDE (2009), Revisión de políticas nacionales de educación: la educación terciaria en Chile 2009, OECD Publishing [DOI: 10.1787/9789264051386].

OCDE (2005), La definición y selección de competencias claves. Resumen ejecutivo, París, OCDE, <www.deseco.admin.ch>. OCDE (2003), Marcos teóricos de PISA 2003. Conocimientos y destrezas en Matemáticas, Lectura, Ciencias y Solución de problemas, París, OCDE.

Palominos, Fredi E., Miguel A. Méndez y Rosa A. Barrera (2014), "Sistema de perfeccionamiento orientado a competencias para docentes de la educación superior", en Formación Universitaria, vol. 7, núm. 3, pp. 11-22.

Paredes, Ricardo, Francisca Bogolasky, Verónica Cabezas, Rosario Rivero y Magdalena Zahri (2013), Los determinantes del primer trabajo para profesores de educación básica en la Región Metropolitana, SANTIAGO DE CHILE,
MINEDUC, Proyecto FONIDE F611105.

Pedraja, Liliana, Carmen Araneda, Emilio Rodríguez y Juan Rodríguez (2012), “ Calidad en la formación inicial docente: evidencia empírica de las universidades chilenas", en Formación Universitaria, vol. 5, núm. 4, pp. 1526.

Person, Ann, Lisbeth Goble y Julie Bruch (2014), Developing competency-based program models in three community colleges, Oakland, Mathematic Policy Research.

Perrenoud, Philippe (2013), Desenvolver competências ou ensinar saberes?'A escola que prepara para a vida, Porto Alegre, Editora Penso.

Poblete, Álvaro y Verónica Díaz (2008), “Integración y contextualización de formas didáctico-pedagógicas y de contenido de un currículo basado en competencias en la formación de estudiantes de Pedagogía en Matemáticas y Computación”, Santiago de Chile, MINEDUC, Proyecto MECESUP ULA0608.

Poblete, Álvaro y Verónica Díaz (2004), "Evaluación de las competencias profesionales del profesor de matemáticas en el marco de la reforma educacional", Santiago de Chile, Comisión Nacional de Investigación Científica y Tecnológica (CONICyT), Proyecto FONDECyT 1010980.

Pochulu, Marcel, Vicenc Font y Mabel Rodríguez (2016), "Desarrollo de la competencia en análisis didáctico de formadores de futuros profesores de matemática a través del diseño de tareas", en Revista Latinoamericana de Investigación en Matemática Educativa, vol. 19, núm. 1, pp. 71-98 [DOI: 10.12802/relime.13.1913].

Porter, Stephen y Kevin Reilly (2014), Competency-based education as a potential strategy to increase learning and lower costs, Washington, HCM Strategists.

Proyecto Tuning América Latina (2007), Reflexiones y perspectivas de la Educación Superior en América Latina. Informe final. Proyecto Tuning América Latina, 20042007, <http://tuning.unideusto.org/tuningal/index. php?option $=$ com $>$.

Riesco, Manuel (2008), "El enfoque por competencias en el EEES y sus implicaciones en la enseñanza y el aprendizaje", en Tendencias Pedagógicas, vol. 13, pp. 79- 
105.

Rodríguez, Emilio, Nicolás Fleet y Milagro Delgado (2009), "La acreditación en la generación de información sobre la calidad de la educación superior", en Calidad en la Educación, vol. 31, pp. 212-230.

Roe, Robert A. (2002), "What makes a competent psychologist?", en European Psychologist, vol. 7, núm. 3, pp. 192-202 [DOI: 10.1027//1016-9040.7.3.192].

Rychen, Dominique S. y Laura H. Salganik (2006), Las competencias clave para el bienestar personal, social y económico, Archidona (Málaga), Ediciones Aljibe.

Schwab, Joseph (1983), "The practical 4: something for curriculum professors to do", en Curriculum Inquiry, vol. 13, pp. 239-266.

Spencer, Lyle M., y Signe M. Spencer (1993), Competence at work. Models for superior performance, Nueva York, Wiley.

Taba, Hilda (1974), La elaboración del currículo, Troquel, Argentina.

Tardif, Jacques (2003), "Développer un programme par compétences: de l'intention à la mise en oeuvre", en Pedagogie Collegiale, vol. 16, núm. 3, pp. 36-44.

Tejada, José (2002), "El docente universitario ante los nuevos escenarios: implicaciones para la innovación docente", en Acción Pedagógica, vol. 11, núm. 2, pp. 30-42.

Tobón, Sergio (2013), Formación integral y competencias, Bogotá, Eco Ediciones.

Tobón, Sergio (2004), Formación basada en competencias, pensamiento complejo, diseño curricular y didáctica, Bogotá, Eco Ediciones.

Tuning Educational Structures in Europe (2006), <http://www.unideusto.org/tuningeu/images / stories/Publications/Tuning_brochure_en_espanol_ listo.pdf>

Tyler, Frederick T. (1960), "Teacher personalities and teaching competencies", en The Social Review, vol. 64, núm. 8, pp. 429-449.

Velasco, Paloma, Begoña Learreta, Claudia Kober e Irene Tan (2014), "Faculty perspective on competency development in higher education: an international study", en Higher Learning Research Communications, vol. 4, núm. 4, pp. 86-100.

Vélez, Griselda y Laura Terán (2009), "Modelos para el diseño curricular", en Pampedia, vol. 6, pp. 55-65.

Verano-Tacoronte, Domingo, Sara González-Betancor, Alicia Bolívar-Cruz, M. Fernández-Monroy e I. Galván-Sánchez (2016), "Valoración de la competencia de comunicación oral de estudiantes universitarios a través de una rúbrica fiable y válida”, en Revista Brasileira de Educação, vol. 21, núm. 64, pp. 39-60 [DOI:10.1590/ S1413-24782016216403].

Villa, Aurelio y Manuel Poblete (2007), Aprendizaje basado en competencias, $1^{\text {a }}$ edición, Bilbao, Ediciones Mensajero.

Walker, Decker F. (1990), Fundamentals of curriculum, San Diego, Harcourt Brace Jovanovich.

Wilson, Patricia, Thomas Cooney y David Stinson (2005), "What constitutes good mathematics teaching and how it develops: nine high school teachers' perspectives", en Fournal of Mathematics Teacher Education, vol. 8, núm. 2, pp. 83-111 [DOI: 10.1007/s10857-005-4796-7].

Zabala, Antoni y Laia Arnau (2007), Cómo aprender y enseñar competencias, Barcelona, Graó.

Zabalza, Miguel Ángel (2012), "Las competencias en la formación del profesorado: de la teoría a las propuestas prácticas”, en Tendencias Pedagógicas, núm. 20, pp. 5-32.

Cómo citar este artículo:

Díaz-Quezada, Verónica, Álvaro Poblete-Letelier y Mónica Gallardo-González (2019), “Rediseño curricular por competencias: experiencia en la formación inicial universitaria en Chile", en Revista Iberoamericana de Educación Superior (RIES), México, UNAM-IISUE/Universia, vol. X, Núm. 27, pp. 72-91, DOI: dx.doi.org/10.22201/ iisue.20072872e.2019.27.341 [consulta: fecha de última consulta]. 